\title{
Mediators of socioeconomic differences in obesity among youth in Ireland and the UK (2011-2021): A Systematic Review Protocol
}

\author{
Authors \\ Mr Thomas Buckley ${ }^{1}$ \\ Dr Frances M. Cronin \\ Ms Delfina Mancebo Guinea Arquez \\ Dr Sinéad Hurley \\ Ms Alice O'Gorman \\ Ms Naeha Lakshmanan \\ Dr Debbi Stanistreet
}

\footnotetext{
${ }^{1}$ Corresponding author; Thomas Buckley, Royal College of Surgeons in Ireland: thomasbuckley@rcsi.ie
} 


\section{Background}

Overweight and obesity is classified as both a global epidemic and a modern-day health crisis and is one of the biggest public health challenges of the $21^{\text {st }}$ century (World Health Organization, 2018, World Health Organization, 2021, Jackson et al., 2020, Ward et al., 2017, Pandita et al., 2016, Ross and Desai, 2014). It is projected that worldwide, obesity and its associated chronic diseases, including diabetes, cardiovascular disease and cancer, will be responsible for up to 92 million premature deaths by 2050 (OECD, 2019). Currently, Ireland is forecast to have one of the highest levels of obesity in Europe, with $43 \%$ of the population projected to be obese $\left(\geq 30 \mathrm{~kg} / \mathrm{m}^{2}\right)$ by 2025 (Pineda et al., 2018). Addressing the rise in the rates of obesity is a recognised priority in the Irish health care system today (HSE, 2020).

Bodyweight is influenced by many factors including diet, physical activity and genetic predisposition (Jackson et al., 2020, Ruiz et al., 2016). However socioeconomic adversity is increasingly being recognised as an important driver in the risk of obesity. Social disturbances throughout childhood are thought to lead to an increase in consumption of junk food as a form of 'self-medication', and a consequent imbalance of energy homeostasis resulting in weight gain and obesity (see Figure 1) (Oliveira, 2020, Hemmingsson, 2018).

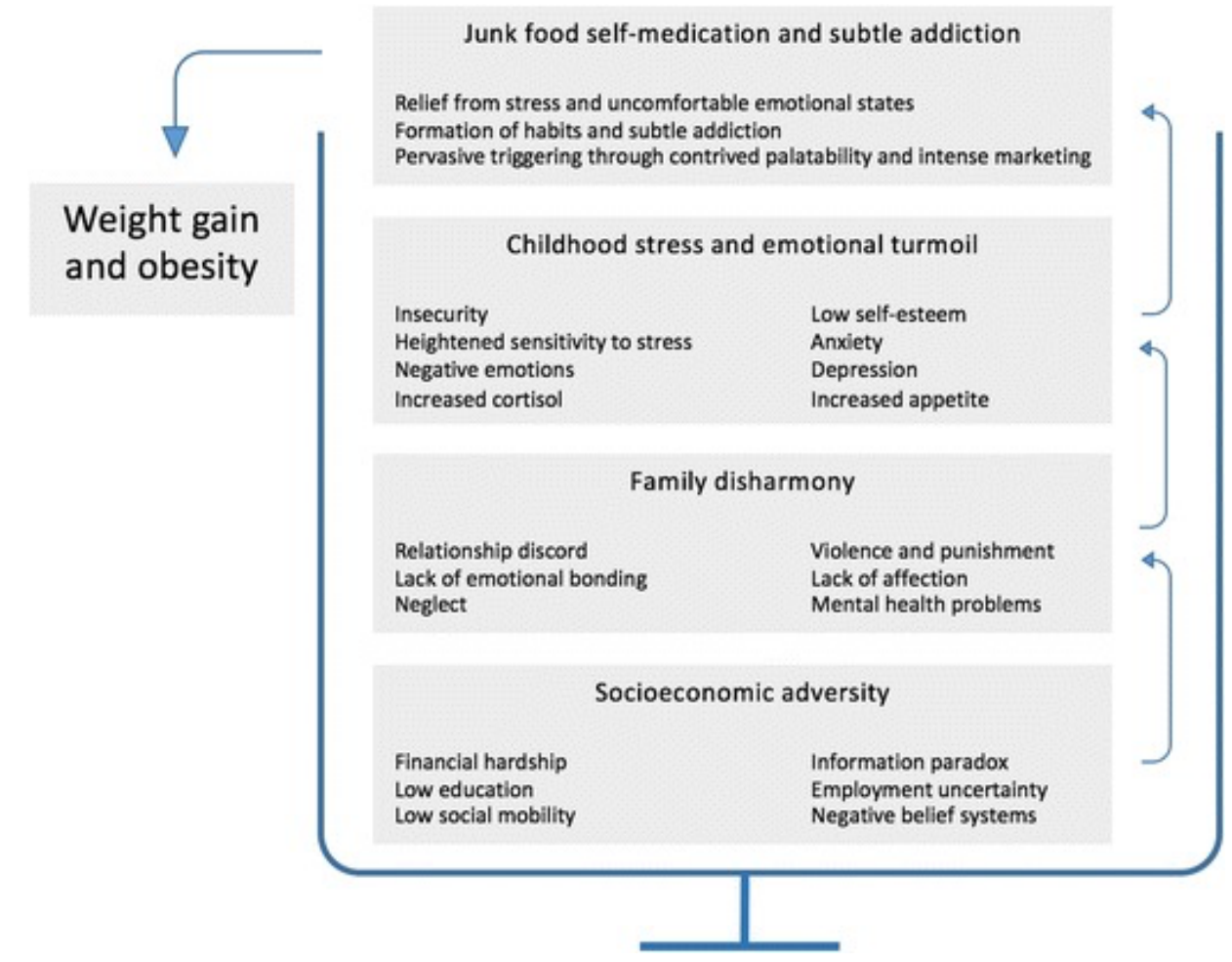

Figure 1 Sequencing and Interaction of obesity risk factors particularly relating to social disturbances during infancy and childhood

Across the world, the healthcare costs associated with obesity are cause for concern. In Ireland in 2009, an estimated $€ 1.1$ billion was spent by the health service as a result of adult obesity alone (Safefood, 2012). By 2015, this spending had increased to over $€ 4.6$ billion, with approximately $€ 1.7$ million in costs attributable to children (Safefood, 2017). An analysis has shown that a $1 \%$ reduction in childhood $\mathrm{BMI}$ would result in a $€ 270$ million saving in annual 
healthcare costs for the Irish government (Safefood, 2017). With current obesity levels as high as $20 \%$ among Irish youth (HSE, 2020), it is clear that interventions to reduce childhood obesity could result in a significant return on investment. However, effective obesity interventions require a thorough understanding of the complex relationship between factors that underlie the cause and progression of obesity in childhood.

Across the OECD it has been recognised that a disproportionate number of children and young people from lower socioeconomic backgrounds live with obesity (HSE, 2016, OECD/European Observatory on Health Systems and Policies, 2019). Historically, a lower socioeconomic position was associated with a lower BMI, however this trend has now reversed with higher rates of obesity being seen in the more deprived groups (Bann, 2018). In Ireland, the use of preventative health care services (annual check-ups, immunization, screening) is much more common among people of a higher socioeconomic position (Social Justice Ireland, 2021). In addition, 93\% of Irish citizens living in the highest income group deem their health as good, compared to only $73 \%$ of people who live in the lowest income quintile (OECD/European Observatory on Health Systems and Policies, 2019). This is perhaps reflected in the finding that the majority of health inequalities between children in Ireland is explained by parentallevel variables, with parental occupation and education, as well as levels of parental smoking and alcohol consumption being among the strongest predictors of childhood obesity (Walsh and Cullinan, 2015).

Addressing childhood obesity is of paramount importance since obesity status persists as the child matures. Around $55 \%$ of obese children will be obese in adolescence, and $80 \%$ of those obese in adolescence will remain obese entering adulthood (Simmonds et al., 2015, Simmonds et al., 2016). Therefore, with childhood and adolescence being crucial life stages that guide health trajectories that might lead to obesity and health inequalities, there is clear evidence that the best approach to long-term, sustainable change in the obesity profile of Ireland is to prevent obesity in early life (HSE, 2016, Herke et al., 2020).

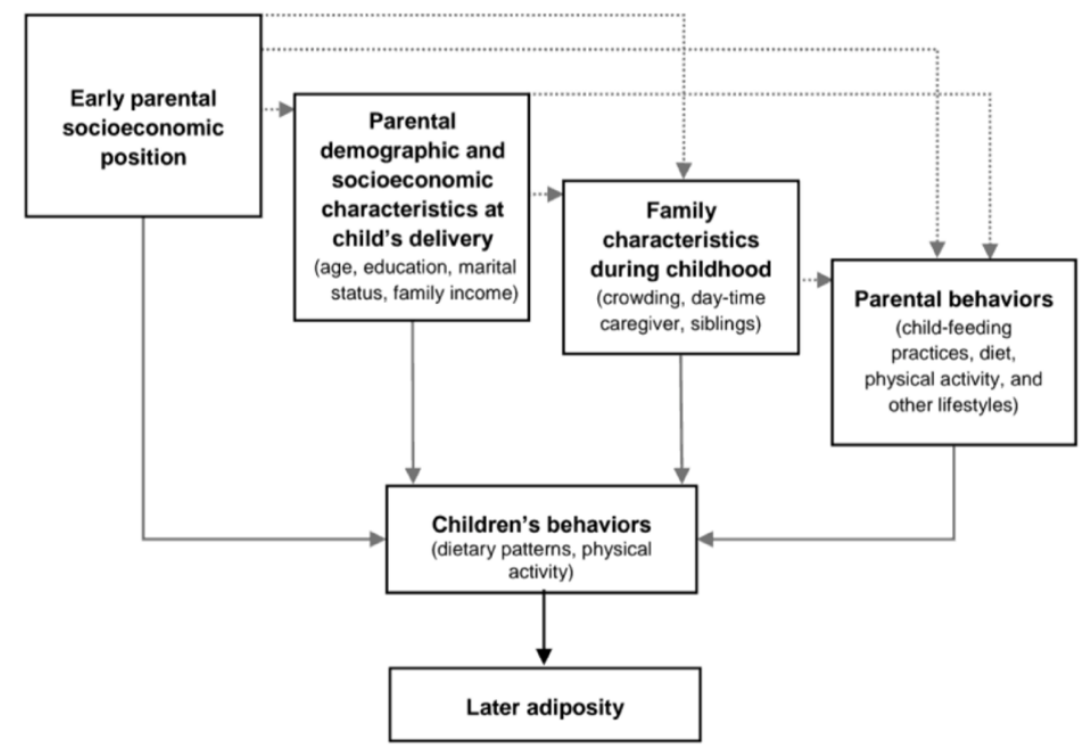

Figure 2 Conceptual framework for examining determinants of children's behaviour and association with later adiposity. Source: (Oliveira, 2020) 
To address the increasing inequalities in childhood obesity outcomes, it is necessary to understand what risk factors are potentially modifiable. A recent review summarized evidence regarding mediators that contribute to the relationship between socioeconomic position and adiposity among youth. Reporting on over 28 studies conducted in OECD countries between 1990 and 2016, a number of modifiable risk factors were identified including: early life experience (including breast feeding, early weaning and maternal smoking in pregnancy), child dietary behaviours (particularly consumption of sugar-sweetened beverages, and eating breakfast), child sedentary activity (particularly television viewing and computer use) and maternal body mass index (Gebremariam et al., 2017).

This systematic review will update and extend Gebremariam's findings with particular focus on research undertaken in Ireland and the United Kingdom. Literature published between 2011 and 2021 will be explored in order to examine extant evidence in relation to factors that explain the relationship between childhood obesity and socioeconomic inequalities.

\section{$\underline{\text { Aim }}$}

The aim of this systematic review is to summarize the existing evidence published between 2011 - 2021, regarding the factors that mediate or contribute to the relationship between socioeconomic position and obesity among youth in Ireland and the UK.

\section{Methods and Design}

A systematic review protocol describes the rationale, hypothesis, and planned methods of the review. To minimize bias, it is vital that methods to be utilized are established in advance of the review process as demonstrated in a systematic review protocol (Lasserson et al.). For example, prior knowledge of the data extraction may influence the review question and eligibility criteria. Protocol publication prior to the review promotes transparency of the systematic review process, reduces the risk of paper duplication, and encourages peer review of the suggested method.

This review will follow the Preferred Reporting Items for Systematic Reviews and MetaAnalysis (PRISMA) checklist

\section{Defining and Aligning the Objective(s) and Question(s)}

To define the review question, an information specialist will conduct an exploratory search of papers across four databases using the key search terms. Databases searched will be: Ovid Medline, Embase, Web of Science and Ebscohost.

\section{Defining and Aligning the Inclusion Criteria with the Objective(s) and Question(s)}

The inclusion and exclusion criteria employed by Gebremariam at al. (Gebremariam et al., 2017) were initially considered, in addition to information from the preliminary abstract screening, and both sources have been adapted to the purposes of the study. The final inclusion and exclusion criteria are as follows: 


\section{Inclusion Criteria}

- Studies exploring mediators of the association between at least one indicator of SEP and at least one indicator of adiposity

- Studies published in English Language

- Studies including youth ( $\leq 18$ years of age)

- Studies conducted in Ireland and the United Kingdom

- Studies published between the years 2011 - 2021

\section{Exclusion Criteria}

- Studies using qualitative methodology

- Studies where analytic methods were not clearly reported

- Studies conducted among clinical populations

- Studies assessing underweight or stunting as an outcome measure

- Studies assessing birth weight as an outcome measure

\section{Describing the planned approach to evidence searching, selection, data extraction and presentation of evidence (Search Strategy)}

The search strategy in this systematic review protocol will be based on the search strategy utilized by Gebremariam et al.(2017).

The keywords used in the preliminary process will be refined after title, abstract and index terms screening of the first 100 of the articles by three researchers. The studies of interest will be those that reported on possible mediators of the association between socioeconomic position and measures of adiposity among youth. Therefore, the 'AND' Boolean operator will be applied to the following groups of keywords:

- Keywords for adiposity (e.g., BMI, Body Weight, Overweight, Adiposity, Obesity)

- Keywords for socioeconomic position (e.g., Socioeconomic, Social Class, Education, Occupation, Income)

- Keywords for mediating factors (e.g., mediat*, attenuat* or indirect)

- Keywords for population of interest (e.g., Infant, Child, Adolescent, Youth)

The search results from each database are reported in Appendix 1.

\section{Searching for the Evidence}

Following the preliminary title and abstract screening, the eligibility criteria will be refined, and the following will be used for the purposes of the review:

- Type of Publication: Journal articles

- Time Frame: 2011 - 2021

- Language: English

- Type of Studies: Studies published in English Language in peer-reviewed journals

- Study Population: Children aged $\leq 18$ years of age living in Ireland and the UK

\section{Selecting the Evidence}


Extracted search results will be stored in Endnote ${ }^{\circledR} 20$ reference manager software, with duplicates noted and removed. The remaining results will be exported to Rayyan ${ }^{\circledR}$, an opensource tool that will be utilized throughout the screening process to identify studies that fit the inclusion and exclusion criteria of this systematic review.

A three-stage strategy will be used to screen the studies in Rayyan ${ }^{\circledR}$. Firstly, three team members will independently screen studies 'blindly' (where no individual has access to another's decisions) on title and abstract to identify studies that fit the inclusion criteria. Second, the full text for the relevant papers will be retrieved and once again screened in a blind fashion to ensure consistency of the process. Third, a final screen will occur at the data extraction point, with the review aiming to reach a $100 \%$ agreement at each stage of the process. Any conflict will be resolved by discussion by the team members, with the final decision being made by the most senior author (DS) if required.

A hand search of reference lists of all literature included in this systematic review will also be conducted to identify any further studies for inclusion.

An outline of the search outcomes, screening and selection process will be reported in a PRISMA flow diagram (Appendix 2).

\section{Extracting the Evidence}

Following the preliminary title and abstract screening two reviewers will utilize the records to validate the data extraction tool. A Microsoft Excel ${ }^{\circledR}$ form will be created, based upon the Gebremariam et al. (Gebremariam et al., 2017) method in order to systematize the data extraction process. The reviewers will adapt the form as required, tailoring the headings based on the research question and objectives as a guide. Where necessary, the rationale for data extraction and any remaining questions regarding the data collection process, will be discussed by an additional member of the team. Any disagreements between reviewers will be resolved by discussion before the Excel ${ }^{\circledR}$ form is completed. This will then be used to populate tables (based on the Gebremariam method) presenting the full set of included studies (Appendix 3).

Two tables will be constructed for the data extraction process of the relevant review papers. The first will include information regarding: (a) author (year) and country (b) sample characteristics ( $n$, age (SD), \% female) (c) study design and instruments (d) indicator of adiposity (including measurement method and categorization (e) indicator of socioeconomic position and ( $f$ ) mediators. The second table will include information regarding: (a) study (b) mediated relationship (direction of the association) (c) method used to assess mediation and (d) mediation results.

\section{Study Quality Assessment}

An adapted version of the Liverpool Quality Assessment Tool (Pope et al., 2013) and the Effective Public Health Practice Project Quality Assessment Tool (Jackson \& Water, 2005) will be utilized for the critical appraisal of each included journal article. After study assessment, an overall quality score will be generated, with scores as follows: 'Strong', 'Moderate' and 
'Weak'. Techniques to be employed for paper scoring include: Selection Procedures (assessing selection bias and validity of methods); Baseline Assessment (assessing differences between selected groups); Outcome Assessment (assessing dropouts and withdrawals); Analysis (assessing confounding variables and statistical methods) and Impact (assessing the study's applicability to this review)

\section{Presentation of the Results}

The 'Preferred Reporting Items for Systematic reviews and Meta-Analyses' (PRISMA) will be employed to ensure the core concepts and key items of this Systematic Review are reported in a transparent manner (Appendix 4).

Summarizing the evidence in relation to the purpose of the review, making conclusions and noting any implications of the findings

A comprehensive summary of the principal findings will be provided. This will summarize the literature published in Ireland and the United Kingdom between 2011 and 2021 regarding factors which mediate or attenuate the socioeconomic differentials found in childhood obesity outcomes. 


\section{References}

BANN, D. 2018. Socioeconomic inequalities in childhood and adolescent body-mass index, weight, and height from 1953 to 2015: an analysis of four longitudinal, observational, British birth cohort studies. The Lancet: Public Health, 3, E194-E203.

GEBREMARIAM, M. K., LIEN, N., NIANOGO, R. A. \& ARAH, O. A. 2017. Mediators of socioeconomic differences in adiposity among youth: a systematic review. Obes Rev, 18, 880-898.

HEMMINGSSON, E. 2018. Early Childhood Obesity Risk Factors: Socioeconomic Adversity, Family Dysfunction, Offspring Distress, and Junk Food Self-Medication. Curr Obes Rep, 7, 204-209.

HERKE, M., MOOR, I., WINTER, K., HOFFMANN, S., SPALLEK, J., HILGER-KOLB, J., PISCHKE, C., DRAGANO, N., NOVELLI, A. \& RICHTER, M. 2020. Role of contextual and compositional characteristics of schools for health inequalities in childhood and adolescence: protocol for a scoping review. BMJ Open, 10, e038999.

HSE. 2016. A Healthy Weight for Ireland: Obesity Policy and Action Plan 2016 - 2025 [Online].

Available: https://www.gov.ie/en/publication/c778a9-a-healthy-weight-for-ireland-obesitypolicy-and-action-plan-2016-202/ [Accessed 28/5/21 2021].

HSE. 2020. National Obesity Clinical Programme [Online]. Available: https://www.hse.ie/eng/about/who/cspd/ncps/obesity/ [Accessed].

JACKSON, N., WATERS, E., GUIDELINES FOR SYSTEMATIC REVIEWS IN HEALTH, P. \& PUBLIC HEALTH, T. 2005. Criteria for the systematic review of health promotion and public health interventions. Health Promot Int, 20, 367-74.

JACKSON, S. E., LLEWELLYN, C. H. \& SMITH, L. 2020. The obesity epidemic - Nature via nurture: A narrative review of high-income countries. SAGE Open Med, 8, 2050312120918265.

LASSERSON, T. J., THOMAS, J., HIGGINS, PTJ. Starting a review [Online]. Cochrane Training. Available: https://training.cochrane.org/handbook/current/chapter-01\#section-1-5 [Accessed].

OECD. 2019. The Heavy Burden of Obesity: The Economics of Prevention [Online]. OECD Publishing, Paris: OECD Helath Policy Studies. Available: https://www.oecdilibrary.org/docserver/67450d67en.pdf?expires $=1624381300 \& i d=i d \& a c c n a m e=$ guest\&checksum $=64$ BB73ABCC9F89B66398 48E40D0726F7 [Accessed].

OECD/EUROPEAN OBSERVATORY ON HEALTH SYSTEMS AND POLICIES 2019. Ireland: Country Health Profile. State of Health in the EU.

OLIVEIRA, A., DURÃO, C., \& LOPES, C. 2020. Social and Health Behaviour Determinants of Obesity. Understanding Obesity: From its Causes to impact on Life, 1, 27-54.

PANDITA, A., SHARMA, D., PANDITA, D., PAWAR, S., TARIQ, M. \& KAUL, A. 2016. Childhood obesity: prevention is better than cure. Diabetes Metab Syndr Obes, 9, 83-9.

PINEDA, E., SANCHEZ-ROMERO, L. M., BROWN, M., JACCARD, A., JEWELL, J., GALEA, G., WEBBER, L. \& BREDA, J. 2018. Forecasting Future Trends in Obesity across Europe: The Value of Improving Surveillance. Obes Facts, 11, 360-371.

POPE, D., IRVING, G., BRUCE, NG., REHFUESS, ER. 2013. Methodological Quality Assessment for Intervention and Observational Studies Using Standardised Instruments: Liverpool Quality Assessment Tools (LQATs) [Online]. [Accessed].

ROSS, M. G. \& DESAI, M. 2014. Developmental programming of appetite/satiety. Ann Nutr Metab, 64 Suppl 1, 36-44.

RUIZ, M., GOLDBLATT, P., MORRISON, J., PORTA, D., FORASTIERE, F., HRYHORCZUK, D., ANTIPKIN, Y., SAUREL-CUBIZOLLES, M. J., LIORET, S., VRIJHEID, M., TORRENT, M., INIGUEZ, C., LARRANAGA, I., BAKOULA, C., VELTSISTA, A., VAN EIJSDEN, M., VRIJKOTTE, 
T. G., ANDRYSKOVA, L., DUSEK, L., BARROS, H., CORREIA, S., JARVELIN, M. R., TAANILA, A., LUDVIGSSON, J., FARESJO, T., MARMOT, M. \& PIKHART, H. 2016. Impact of Low Maternal Education on Early Childhood Overweight and Obesity in Europe. Paediatr Perinat Epidemiol, 30, 274-84.

SAFEFOOD. 2012. Annual Report 2012 [Online]. Available:

https://www.safefood.net/getattachment/1bd10604-b95b-4e75-8730-

183747af7e1a/safefood-Annual-Report-2012.pdf?lang=en-IE [Accessed].

SAFEFOOD. 2017. What are the estimated costs of childhood overweight and obesity on the island of Ireland? [Online]. Available: https://www.safefood.net/getmedia/07c9ceb0-dd71-4658b679-cfc40c03e16b/Cost-of-childhood-obesity-Report.aspx?ext=.pdf [Accessed].

SIMMONDS, M., BURCH, J., LLEWELLYN, A., GRIFFITHS, C., YANG, H., OWEN, C., DUFFY, S. \& WOOLACOTT, N. 2015. The use of measures of obesity in childhood for predicting obesity and the development of obesity-related diseases in adulthood: a systematic review and meta-analysis. Health Technol Assess, 19, 1-336.

SIMMONDS, M., LLEWELLYN, A., OWEN, C. G. \& WOOLACOTT, N. 2016. Predicting adult obesity from childhood obesity: a systematic review and meta-analysis. Obes Rev, 17, 95-107.

SOCIAL JUSTICE IRELAND. 2021. Health Inequalities - the postcode lottery [Online]. Available: https://www.socialjustice.ie/content/policy-issues/health-inequalities-postcode-lottery [Accessed].

WALSH, B. \& CULLINAN, J. 2015. Decomposing socioeconomic inequalities in childhood obesity: evidence from Ireland. Econ Hum Biol, 16, 60-72.

WARD, Z. J., LONG, M. W., RESCH, S. C., GILES, C. M., CRADOCK, A. L. \& GORTMAKER, S. L. 2017. Simulation of Growth Trajectories of Childhood Obesity into Adulthood. N Engl J Med, 377, 2145-2153.

WORLD HEALTH ORGANIZATION. 2018. Taking Action on Childhood Obesity [Online]. Available: https://apps.who.int/iris/bitstream/handle/10665/274792/WHO-NMH-PND-ECHO-18.1eng.pdf [Accessed].

WORLD HEALTH ORGANIZATION. 2021. Obesity and Overweight [Online]. Available: https://www.who.int/news-room/fact-sheets/detail/obesity-and-overweight [Accessed]. 
Appendix 1 Search terms and search summary adoption

\begin{tabular}{|l|l|c|}
\hline & $\begin{array}{l}\text { Ovid MEDLINE(R) and Epub Ahead of Print, In-Process, In-Data-Review \& } \\
\text { Other Non-Indexed Citations, Daily and Versions(R) 1946 to May 11 2021 }\end{array}$ & \\
\hline 1 & $\begin{array}{l}\text { adiposity.mp. or exp Adiposity/ or *Body Weight/ or exp Obesity/ or } \\
\text { obesity.mp. or overweight.mp. or exp Overweight/ or body mass index.mp. } \\
\text { or exp Body Mass Index/ }\end{array}$ & 299162 \\
\hline 2 & $\begin{array}{l}\text { exp Socioeconomic Factors/ or socioeconomic.mp. or education.mp. or } \\
\text { occupation.mp. or income.mp. }\end{array}$ & 1436516 \\
\hline 3 & \begin{tabular}{l} 
(mediat* or attenuat* or indirect).mp \\
\hline 4
\end{tabular} & $\begin{array}{l}\text { infant/ or infant.mp. or infants.mp.or exp Child/ or (child or children).mp. or } \\
\text { exp Adolescent/ OR adolescent*.mp. or (youth or youths).mp. }\end{array}$ \\
\hline 5 & $\begin{array}{l}\text { ireland or england or wales or scotland or uk or (united adj1 kingdom) or } \\
\text { (great adj1 britain) }\end{array}$ & 4087920 \\
\hline 6 & 1 AND 2 AND 3 AND 4 AND 5 & 206715 \\
\hline 7 & Limit 6 to 2011-2021 & 163 \\
\hline & \multicolumn{1}{|l}{} \\
\hline
\end{tabular}

\begin{tabular}{|l|l|c|}
\hline & EMBASE & \\
\hline $\mathbf{1}$ & $\begin{array}{l}\text { Adiposity:ti,ab,kw or 'Adiposity'/exp or 'Obesity'/exp or obesity:ti,ab,kw or } \\
\text { overweight:ti,ab,kw or 'body weight':ti,ab,kw or 'body mass'/exp or 'body } \\
\text { mass index':ti,ab,kw }\end{array}$ & 1192540 \\
\hline 2 & $\begin{array}{l}\text { 'socioeconomics'/exp or socioeconomic:ti,ab,kw or education:ti,ab,kw or } \\
\text { occupation:ti,ab,kw or income:ti,ab,kw }\end{array}$ & 1180111 \\
\hline 3 & $\begin{array}{l}\text { (mediat* or attenuat* or indirect):ti,ab, de,kw } \\
\text { infant*:ti,ab,kw OR 'child'/exp OR child:ti,ab,de,kw OR children:ti,ab,de,kw } \\
\text { OR 'adolescent'/exp OR adolescent*:ti,ab,kw OR youth*:ti,ab,kw }\end{array}$ & 2310622 \\
\hline 5 & $\begin{array}{l}\text { ireland or england or wales or scotland or uk or (united NEXT/1 kingdom) or } \\
\text { (great NEXT/1 britain) }\end{array}$ & 9449517 \\
\hline 6 & 1 AND 2 AND 3 AND 4 AND 5 & 6495165 \\
\hline 7 & Limit 6 2011-2021 & 518 \\
\hline & \multicolumn{1}{|l}{} \\
\hline
\end{tabular}

\begin{tabular}{|l|l|c|}
\hline & Web of Science Science and Social Science Citation Indexes & \\
\hline $\mathbf{1}$ & TS=(adiposity or obesity or (body NEAR/1 mass)) & 523537 \\
\hline 2 & TS=(socioeconomic or education or occupation or income) & 1081595 \\
\hline 3 & TS=(mediat* or attenuat* or indirect) & 2247971 \\
\hline 4 & TS=(Infant or infants or child or children or adolescent* or youth or youths) & 2082135 \\
\hline 5 & $\begin{array}{l}\text { TS=(ireland or england or wales or scotland or uk or (united NEAR/1 } \\
\text { kingdom) or (great NEAR/1 britain)) }\end{array}$ & 635573 \\
\hline 6 & 1 AND 2 AND 3 AND 4 AND 5 & 61 \\
\hline 7 & Limit 6 2011-2021 & 53 \\
\hline & \multicolumn{2}{|l}{} \\
\hline
\end{tabular}




\begin{tabular}{|l|l|c|}
\hline & APA PSYCHINFO on Ebscohost & \\
\hline $\mathbf{1}$ & $\begin{array}{l}\text { Adiposity or obesity or MM "Obesity" OR MM “body fat" or 'body mass' or } \\
\text { MM "Body Mass Index" }\end{array}$ & 65294 \\
\hline 2 & $\begin{array}{l}\text { DE "Socioeconomic Status" OR DE "Family Socioeconomic Level" OR DE } \\
\text { "Income Level" OR DE "Lower Class" OR DE "Social Class" OR socioeconomic } \\
\text { or education or occupation or income }\end{array}$ & 883738 \\
\hline 3 & TX (mediat* or attenuat* or indirect) & 286005 \\
\hline 4 & Infant or infants or child or children or adolescent* or youth or youths & 1173254 \\
\hline 5 & $\begin{array}{l}\text { ireland or england or wales or scotland or uk or (united N1 kingdom) or } \\
\text { (great N1 britain) }\end{array}$ & 490451 \\
\hline 6 & 1 AND 2 AND 3 AND 4 AND 5 & 90 \\
\hline 7 & Limit 6 2011-2021 & 59 \\
\hline & \multicolumn{2}{|l}{} \\
\hline
\end{tabular}




\section{Appendix 2 Prisma Flow Diagram}

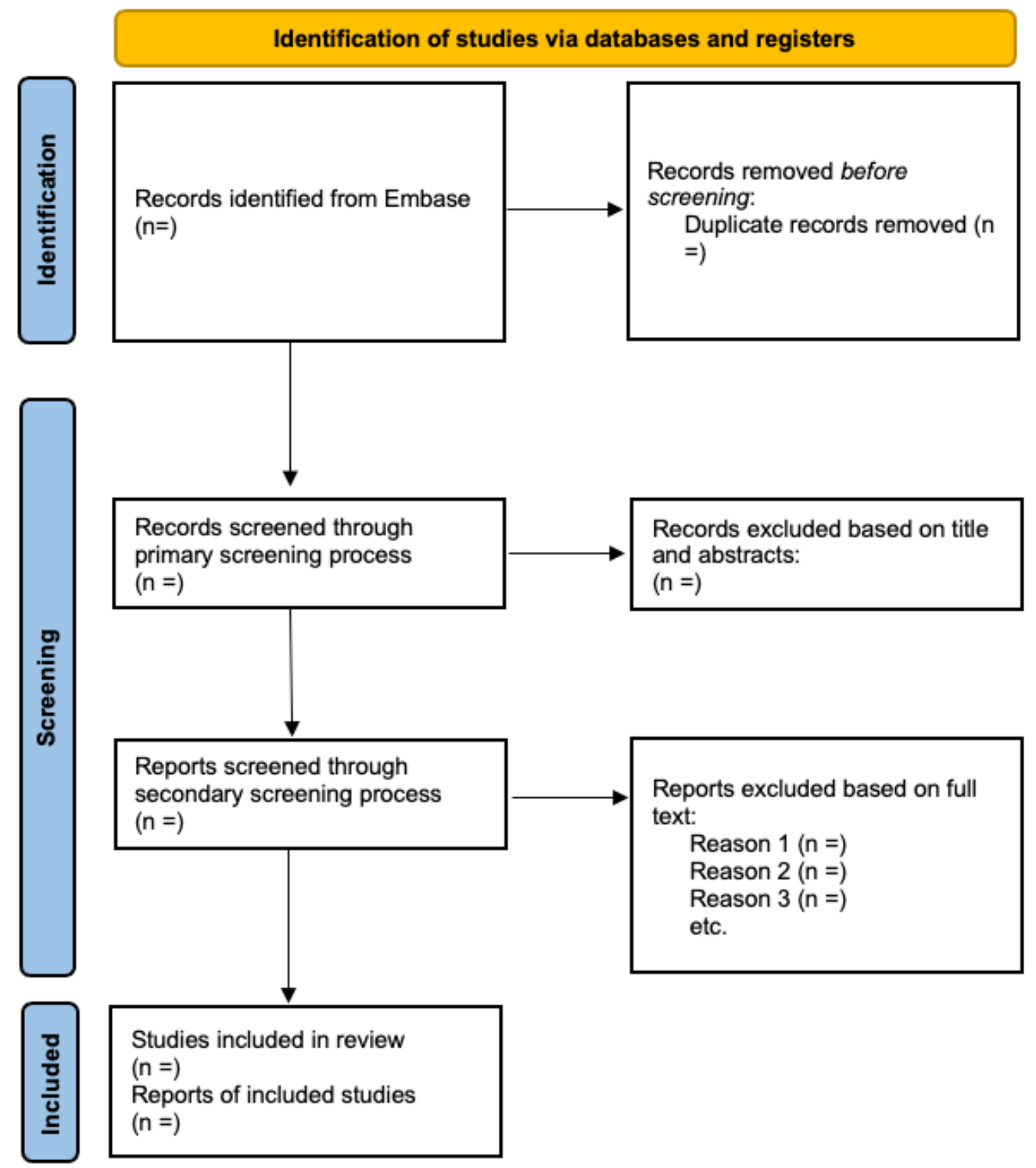

Figure 3 Source: Page MJ, McKenzie JE, Bossuyt PM, Boutron I, Hoffmann TC, Mulrow CD, et al. The PRISMA 2020 statement: an updated guideline for reporting systematic reviews. BMJ 2021;372:n71. doi: 10.1136/bmj.n71 
Appendix 3 Sample Data Extraction Form

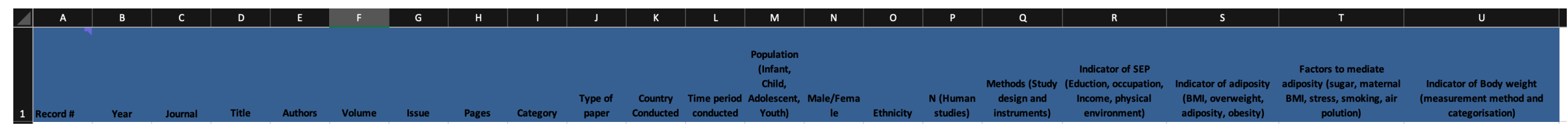

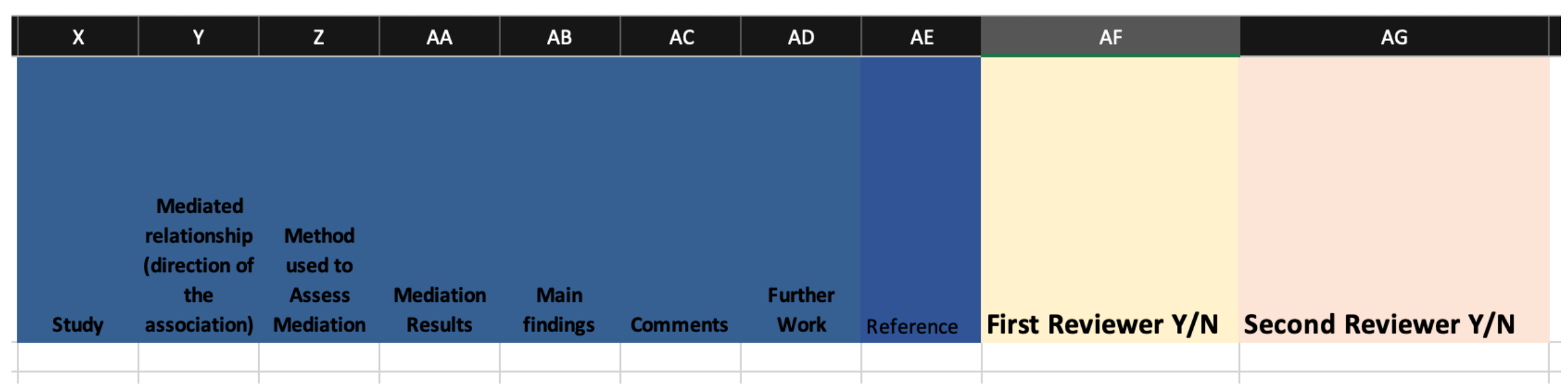




\section{Appendix 4 - Preferred Reporting Items for Systematic reviews and Meta-Analyses Checklist}

\begin{tabular}{|c|c|c|c|}
\hline $\begin{array}{l}\text { Section and } \\
\text { Topic }\end{array}$ & $\begin{array}{l}\text { Item } \\
\#\end{array}$ & Checklist item & $\begin{array}{l}\text { Location } \\
\text { where item } \\
\text { is reported }\end{array}$ \\
\hline \multicolumn{4}{|l|}{ TITLE } \\
\hline Title & 1 & Identify the report as a systematic review. & \\
\hline \multicolumn{4}{|l|}{ ABSTRACT } \\
\hline Abstract & 2 & See the PRISMA 2020 for Abstracts checklist. & \\
\hline \multicolumn{4}{|l|}{ INTRODUCTION } \\
\hline Rationale & 3 & Describe the rationale for the review in the context of existing knowledge. & \\
\hline Objectives & 4 & Provide an explicit statement of the objective(s) or question(s) the review addresses. & \\
\hline \multicolumn{4}{|l|}{ METHODS } \\
\hline Eligibility criteria & 5 & Specify the inclusion and exclusion criteria for the review and how studies were grouped for the syntheses. & \\
\hline $\begin{array}{l}\text { Information } \\
\text { sources }\end{array}$ & 6 & $\begin{array}{l}\text { Specify all databases, registers, websites, organisations, reference lists and other sources searched or consulted to identify studies. Specify the } \\
\text { date when each source was last searched or consulted. }\end{array}$ & \\
\hline Search strategy & 7 & Present the full search strategies for all databases, registers and websites, including any filters and limits used. & \\
\hline Selection process & 8 & $\begin{array}{l}\text { Specify the methods used to decide whether a study met the inclusion criteria of the review, including how many reviewers screened each record } \\
\text { and each report retrieved, whether they worked independently, and if applicable, details of automation tools used in the process. }\end{array}$ & \\
\hline $\begin{array}{l}\text { Data collection } \\
\text { process }\end{array}$ & 9 & $\begin{array}{l}\text { Specify the methods used to collect data from reports, including how many reviewers collected data from each report, whether they worked } \\
\text { independently, any processes for obtaining or confirming data from study investigators, and if applicable, details of automation tools used in the } \\
\text { process. }\end{array}$ & \\
\hline \multirow[t]{2}{*}{ Data items } & 10a & $\begin{array}{l}\text { List and define all outcomes for which data were sought. Specify whether all results that were compatible with each outcome domain in each } \\
\text { study were sought (e.g. for all measures, time points, analyses), and if not, the methods used to decide which results to collect. }\end{array}$ & \\
\hline & $10 \mathrm{~b}$ & $\begin{array}{l}\text { List and define all other variables for which data were sought (e.g. participant and intervention characteristics, funding sources). Describe any } \\
\text { assumptions made about any missing or unclear information. }\end{array}$ & \\
\hline $\begin{array}{l}\text { Study risk of bias } \\
\text { assessment }\end{array}$ & 11 & $\begin{array}{l}\text { Specify the methods used to assess risk of bias in the included studies, including details of the tool(s) used, how many reviewers assessed each } \\
\text { study and whether they worked independently, and if applicable, details of automation tools used in the process. }\end{array}$ & \\
\hline Effect measures & 12 & Specify for each outcome the effect measure(s) (e.g. risk ratio, mean difference) used in the synthesis or presentation of results. & \\
\hline \multirow[t]{6}{*}{$\begin{array}{l}\text { Synthesis } \\
\text { methods }\end{array}$} & 13a & $\begin{array}{l}\text { Describe the processes used to decide which studies were eligible for each synthesis (e.g. tabulating the study intervention characteristics and } \\
\text { comparing against the planned groups for each synthesis (item \#5)). }\end{array}$ & \\
\hline & $13 \mathrm{~b}$ & $\begin{array}{l}\text { Describe any methods required to prepare the data for presentation or synthesis, such as handling of missing summary statistics, or data } \\
\text { conversions. }\end{array}$ & \\
\hline & $13 \mathrm{c}$ & Describe any methods used to tabulate or visually display results of individual studies and syntheses. & \\
\hline & 13d & $\begin{array}{l}\text { Describe any methods used to synthesize results and provide a rationale for the choice(s). If meta-analysis was performed, describe the } \\
\text { model(s), method(s) to identify the presence and extent of statistical heterogeneity, and software package(s) used. }\end{array}$ & \\
\hline & $13 \mathrm{e}$ & Describe any methods used to explore possible causes of heterogeneity among study results (e.g. subgroup analysis, meta-regression). & \\
\hline & $13 f$ & Describe any sensitivity analyses conducted to assess robustness of the synthesized results. & \\
\hline $\begin{array}{l}\text { Reporting bias } \\
\text { assessment }\end{array}$ & 14 & Describe any methods used to assess risk of bias due to missing results in a synthesis (arising from reporting biases). & \\
\hline $\begin{array}{l}\text { Certainty } \\
\text { assessment }\end{array}$ & 15 & Describe any methods used to assess certainty (or confidence) in the body of evidence for an outcome. & \\
\hline
\end{tabular}


continued.../

\begin{tabular}{|c|c|c|c|}
\hline $\begin{array}{l}\text { Section and } \\
\text { Topic }\end{array}$ & Item & Checklist item & $\begin{array}{l}\text { Location } \\
\text { where item } \\
\text { is reported }\end{array}$ \\
\hline \multicolumn{4}{|l|}{ RESULTS } \\
\hline \multirow[t]{2}{*}{ Study selection } & $16 \mathrm{a}$ & $\begin{array}{l}\text { Describe the results of the search and selection process, from the number of records identified in the search to the number of studies included in } \\
\text { the review, ideally using a flow diagram. }\end{array}$ & \\
\hline & $16 \mathrm{~b}$ & Cite studies that might appear to meet the inclusion criteria, but which were excluded, and explain why they were excluded. & \\
\hline $\begin{array}{l}\text { Study } \\
\text { characteristics }\end{array}$ & 17 & Cite each included study and present its characteristics. & \\
\hline $\begin{array}{l}\text { Risk of bias in } \\
\text { studies }\end{array}$ & 18 & Present assessments of risk of bias for each included study. & \\
\hline \begin{tabular}{|l|l|} 
Results of \\
individual studies
\end{tabular} & 19 & $\begin{array}{l}\text { For all outcomes, present, for each study: (a) summary statistics for each group (where appropriate) and (b) an effect estimate and its precision } \\
\text { (e.g. confidence/credible interval), ideally using structured tables or plots. }\end{array}$ & \\
\hline \multirow{4}{*}{$\begin{array}{l}\text { Results of } \\
\text { syntheses }\end{array}$} & $20 \mathrm{a}$ & For each synthesis, briefly summarise the characteristics and risk of bias among contributing studies. & \\
\hline & $20 \mathrm{~b}$ & $\begin{array}{l}\text { Present results of all statistical syntheses conducted. If meta-analysis was done, present for each the summary estimate and its precision (e.g. } \\
\text { confidence/credible interval) and measures of statistical heterogeneity. If comparing groups, describe the direction of the effect. }\end{array}$ & \\
\hline & $20 \mathrm{c}$ & Present results of all investigations of possible causes of heterogeneity among study results. & \\
\hline & $20 \mathrm{~d}$ & Present results of all sensitivity analyses conducted to assess the robustness of the synthesized results. & \\
\hline Reporting biases & 21 & Present assessments of risk of bias due to missing results (arising from reporting biases) for each synthesis assessed. & \\
\hline $\begin{array}{l}\text { Certainty of } \\
\text { evidence }\end{array}$ & 22 & Present assessments of certainty (or confidence) in the body of evidence for each outcome assessed. & \\
\hline \multicolumn{4}{|l|}{ DISCUSSION } \\
\hline \multirow[t]{4}{*}{ Discussion } & 23a & Provide a general interpretation of the results in the context of other evidence. & \\
\hline & $23 \mathrm{~b}$ & Discuss any limitations of the evidence included in the review. & \\
\hline & $23 \mathrm{c}$ & Discuss any limitations of the review processes used. & \\
\hline & 23d & Discuss implications of the results for practice, policy, and future research. & \\
\hline \multicolumn{4}{|c|}{ OTHER INFORMATION } \\
\hline \multirow{3}{*}{$\begin{array}{l}\text { Registration and } \\
\text { protocol }\end{array}$} & $24 a$ & Provide registration information for the review, including register name and registration number, or state that the review was not registered. & \\
\hline & $24 \mathrm{~b}$ & Indicate where the review protocol can be accessed, or state that a protocol was not prepared. & \\
\hline & $24 \mathrm{c}$ & Describe and explain any amendments to information provided at registration or in the protocol. & \\
\hline Support & 25 & Describe sources of financial or non-financial support for the review, and the role of the funders or sponsors in the review. & \\
\hline $\begin{array}{l}\text { Competing } \\
\text { interests }\end{array}$ & 26 & Declare any competing interests of review authors. & \\
\hline $\begin{array}{l}\text { Availability of } \\
\text { data, code and } \\
\text { other materials }\end{array}$ & 27 & $\begin{array}{l}\text { Report which of the following are publicly available and where they can be found: template data collection forms; data extracted from included } \\
\text { studies; data used for all analyses; analytic code; any other materials used in the review. }\end{array}$ & \\
\hline
\end{tabular}

Figure 4 Source: Page MJ, McKenzie JE, Bossuyt PM, Boutron I, Hoffmann TC, Mulrow CD, et al. The PRISMA 2020 statement: an updated guideline

for reporting systematic reviews. BMJ 2021;372:n71. doi: 10.1136/bmj.n71 